\title{
Spherical Casimir pistons
}

\author{
J.S.Dowker ${ }^{1}$ \\ Theory Group, \\ School of Physics and Astronomy, \\ The University of Manchester, \\ Manchester, England
}

\begin{abstract}
A piston is introduced into a spherical lune Casimir cavity turning it into two adjacent lunes separated by the (hemispherical) piston. On the basis of zeta-function regularisation, the vacuum energy of the arrangement is finite for conformal propagation in space-time. For even spheres this energy is independent of the angle of the lune. For odd dimensions it is shown that for all Neumann, or all Dirichlet, boundary conditions the piston is attracted or repelled by the nearest wall if $d=3,7, \ldots$ or if $d=1,5, \ldots$, respectively. For hybrid $N-D$ conditions these requirements are switched. If a mass is added, divergences arise which render the model suspect. The analysis, however, is relatively straightforward and involves the Barnes zeta function. The extension to finite temperatures is made and it is shown that for the $3,7, \ldots$ series of odd spheres, the repulsion by the walls continues but that, above a certain temperature, the free energy acquires two minima symmetrically placed about the mid point.
\end{abstract}

\footnotetext{
${ }^{1}$ dowker@man.ac.uk
} 


\section{Introduction.}

The Casimir piston, [1], has been the subject of considerable investigation, the object being to elucidate the nature of the Casimir effect after rendering some unwelcome infinities irrelevant from the outset. I refer only to the two recent works by Kirsten and Fulling, [2] and by Fucci and Kirsten, [3], where more extensive references and background information can be found.

The basic situation reflects Casimir's original question concerning the global effect of introducing a partition into a cavity, $\mathcal{M}^{\prime}$, so that $\mathcal{M}^{\prime}$ becomes the union of two (joined) pieces, $\mathcal{M} \cup \mathcal{M}^{*}$.

Working with the free energy, $F$, this effect is expressed as the difference before and after constructing the partition:

$$
\Delta F=F(\mathcal{M})+F\left(\mathcal{M}^{*}\right)-F\left(\mathcal{M}^{\prime}\right),
$$

which was referred to as 'Casimir renormalisation' in [4]. As remarked in this reference, if the partition were flexible, or free, it would seek to adjust itself so as to minimise $\Delta F$.

The subtraction in (1) is not the same as that employed in [1], but has the same consequence. If the cavity, $\mathcal{M}^{\prime}$, is considered to sit inside a larger enclosure (the universe), equation (1) for the internal quantities follows because the exterior contributions to the before and after free energies cancel, being independent of the partitioning. Furthermore, the last term in (1) is also independent of the partition and can be dropped when, say, evaluating the force on the partition. I thus regain the expression used in [1], and later works.

At zero temperature, Lukosz, [5], uses a similar technique and I shall here mainly restrict myself to this case when $F$ becomes $E$, the vacuum energy. Comments on finite temperature effects are made in a later section.

There are a number of arrangements of this partitioning, that described in [1] being the most common, a transverse, movable flat plate (the piston) in a rectangular box in flat space. By contrast, Fucci and Kirsten, [3], consider, as an example, a curved space situation where the manifold, $\mathcal{M}^{\prime}$, is a (compressed) ball and the piston is an interior sphere enclosing a global monopole.

Here, I wish to describe another example involving a curved space. I will not answer any outstanding questions, nor will the system be particularly realisable, but it has the advantage of a simple analysis, yielding closed formulae. One does not encounter the Epstein $\zeta$-functions arising in rectangular geometries, e.g. $[4,6]$ and the many later discussions, which must be something of a novelty in this topic. 


\section{A curved piston}

My model takes space-time (the universe) as ultra static, of the form $T \times \widetilde{S}^{d}$ where $\widetilde{S}^{d}$ is a $d$-dimensional sphere whose azimuthal angle, $\phi$, has been unrolled so that $0 \leq \phi \leq \infty$. The cavity, $\mathcal{M}^{\prime}$, is obtained by choosing $(d-1)$-hemispherical boundaries at, say, the azimuthal angles $\phi=0$ and $\phi=\alpha$ making it a $d$-lune, $\mathrm{S}_{\alpha}^{d}$, of angle $\alpha$. I consider a scalar field in this cavity subject to Dirichlet or Neumann conditions at the boundary. The rest of the $d$-sphere, another lune, of infinite angle, constitutes the 'outside' region. ${ }^{2}$ These manifolds are singular.

A piston ${ }^{3}$ is now introduced in $\mathcal{M}^{\prime}$ as another hemisphere located at the (variable) angle $\phi=\beta<\alpha$, again with $D$ or $N$ conditions. This makes the manifolds $\mathcal{M}, \mathcal{M}^{*}$, lunes of angles $\beta$ and $\alpha-\beta$ respectively. There is nothing, in this model, to stop $\alpha$ becoming infinitely large, sending one wall to infinity.

All one needs to implement the Casimir renormalisation, (1), is the vacuum energy on a $D$ or $N$ lune.

\section{Vacuum energy on lunes. Conformal coupling.}

It is a standard result, e.g. [4,6], that, if it is not infinite, the vacuum energy of a scalar field on an ultra static space time is given by,

$$
E=\frac{1}{2} \zeta_{\mathcal{M}}(-1 / 2)
$$

in terms of the $\zeta$-function on the spatial section, $\mathcal{M}$, defined by

$$
\zeta_{\mathcal{M}}(s)=\sum \frac{1}{\lambda_{n}^{s}},
$$

where $\lambda_{n}$ are the eigenvalues of the spatial part of the propagation operator. This part generally takes the form of the Laplacian, or improved Laplacian, on $\mathcal{M}$.

For simplicity, I assume that the scalar field is massless and is conformally coupled in $d+1$ dimensions. This has the highly simplifying consequence of making the eigenvalues on the lune perfect squares, determined by,

$$
\sqrt{\lambda_{n}}=a+\frac{\pi}{\beta} n_{1}+n_{2}+\ldots+n_{d}
$$

\footnotetext{
2 If one wished, the universe could be made finite by the introduction of a far boundary at some large value of $\phi$ or the unrolled sphere could be replaced by an ordinary one. All angles would then lie between 0 and $2 \pi$.

3 The word 'piston' is, perhaps, slightly inaccurate.
} 
where $\beta$ is the angle of the lune, and the $n_{i}$ are non-negative integers. The constant $a$ depends on the boundary conditions, Neumann conditions corresponding to $a=$ $a_{N} \equiv(d-1) / 2$ and Dirichlet to $a=a_{D} \equiv \beta / \pi+(d-1) / 2 .$.

These eigenvalues follow by standard separation of variables. Gromes, [7], gives the analysis for the two-lune (see also, earlier, Pockels, [8] pp.100-109). The higher lune result reflects the nesting of their metrics. The resulting $\zeta$-function from (3) is a Barnes $\zeta$-function.

The situation is exactly the same as that encountered in [9] except that there the lune is a special one with $\beta=\pi / q, q \in \mathbb{Z}$. The vacuum energies can then be read off from the expressions in [9] because the particular analysis extends to arbitrary $\beta$.

For completeness, I give some appropriate formulae. The $\zeta$-functions (for conformal coupling in $d+1$ dimensions) are

$$
\begin{aligned}
& \zeta_{N}(s)=\zeta_{d}(2 s,(d-1) / 2 \mid \pi / \beta, \mathbf{1}) \\
& \zeta_{D}(s)=\zeta_{d}(2 s, \pi / \beta+(d-1) / 2 \mid \pi / \beta, \mathbf{1})
\end{aligned}
$$

in terms of the Barnes $\zeta$-function, $\zeta_{d}$. Looking at (2), one needs the value of the Barnes function at -1 , and Barnes himself, [10], gives this as a generalised Bernoulli polynomial. The expression for the vacuum energies on lunes is,

$$
E(\beta)=-(\mp)^{d+1} \frac{\beta}{2 \pi(d+1) !} B_{d+1}^{(d)}((d-1) / 2 \mid \pi / \beta, \mathbf{1}) .
$$

The upper sign is for Neumann and the lower one for Dirichlet conditions. Incidentally, adding the $N$ and $D$ values gives the energy on a periodic lune of angle $2 \beta$ and it can be seen that this vanishes for even $d$, as is well known for the full sphere (for conformal coupling).

Explicit computation of the polynomial in (6) reveals that $E(\beta)$ is independent of $\beta$ for even $d$. This can be shown analytically as follows.

By applying Barnes' derivative formula,

$$
\begin{aligned}
\frac{\partial}{\partial q} \frac{1}{q} B_{k}^{(d)}(a \mid q, \boldsymbol{\omega}) & =-\frac{1}{q^{2}} B_{k}^{(d+1)}(a+q \mid q, q, \boldsymbol{\omega}) \\
& =-(-1)^{k} \frac{1}{q^{2}} B_{k}^{(d+1)}(d-1-a+q \mid q, q, \boldsymbol{\omega}),
\end{aligned}
$$

I find

$$
\begin{aligned}
\frac{\partial}{\partial q} \frac{1}{q} B_{d+1}^{(d)}((d-1) / 2 \mid q, \mathbf{1}) & =-\frac{1}{q^{2}} B_{d+1}^{(d+1)}((d-1) / 2+q \mid q, q, \mathbf{1}) \\
& =(-1)^{d} \frac{1}{q^{2}} B_{d+1}^{(d+1)}((d-1) / 2+q \mid q, q, \mathbf{1})
\end{aligned}
$$


which therefore vanishes if $d$ is even.

This result means of course that there is no force (torque) on the internal partition for even $d$.

Turning now to the more interesting case of odd $d$, I give two examples of the rational functions for the internal vacuum energy on an odd $D$ or $N$ lune of angle $\beta=\pi / q$,

$$
\begin{aligned}
E(\beta) & =\frac{q^{4}+5 q^{2}-3}{1440 q}, \quad d=3 \\
& =\frac{2 q^{6}+14 q^{4}+77 q^{2}-62}{120960 q}, \quad d=5 .
\end{aligned}
$$

The Casimir renormalised internal energy of the partitioned manifold is

$$
E(\beta, \alpha)=E(\beta)+E(\alpha-\beta)-E(\alpha)
$$

As a function of $\beta, E(\beta, \alpha)$ is symmetric about the middle point, $\beta=\alpha / 2$, where, therefore, it has an extremum. If a minimum (stability), the partition is repelled by the nearest wall, while if a maximum (instability), it is attracted. Repulsion is more interesting.

Calculation shows that (9) has a minimum for $d=3,7,11, \ldots$ and a maximum for $d=1,5,9,13, \ldots$. I obtain this behaviour analytically in Appendix 1. A roughly similar alternating behaviour is found by Fucci and Kirsten, [3], in a quite different setup, for Dirichlet conditions (but not, curiously, for Neumann ones).

Sending one wall to infinity $\alpha \rightarrow \infty$ while keeping $\beta$ finite does not alter these

conclusions since one is working on the left-hand side of the energy (potential) curve.

\section{Hybrid boundary conditions}

One can ring the changes on boundary conditions. For example, still retaining symmetry, Neumann conditions could be enforced at the outer walls, with Dirichlet at the piston.

The energy of an $N D$ lune is given in terms of that on a $D D$, or $N N$, one, (6), by $(e . g \cdot[11])$,

$$
E_{N D}(\beta)=E(2 \beta)-E(\beta),
$$

and, following the analysis through, the dimension dependence of a maximum or a minimum at the mid point is switched so that, this time, one has a maximum for 
$d=3,7,11, \ldots$ and a minimum for $d=1,5,9,13, \ldots$. For even $d$, the $N D$ energy is zero.

In dimension $d=1$, the consequent repulsion agrees with an old result, discussed and extended by Fulling, Kaplan and Wilson, [12].

\section{Finite temperature}

Another consequence of the simple form of the eigenvalues is that the finite temperature quantities are given as simple series, easily computed. For example the free energy at temperature $T$ is, [9], on the lune of angle $\beta$,

$$
F(T, \beta)=E(\beta)-\frac{T}{2^{d}} \sum_{m=1}^{\infty} \frac{1}{m}\left(\operatorname{coth} \frac{m \pi}{2 \beta T} \pm 1\right) \operatorname{cosech}^{d-1} \frac{m}{2 T} .
$$

As $T$ increases, any minimum at the mid point will, at some point, become a maximum. This is best seen from the high temperature expansion of the free energy given in [4] and [13]. When computing the subtracted quantity, (1), the first two terms in this series, which are proportional to the measure of the domain concerned and of its boundary, respectively, cancel or are constant. The third term is the active one. It is proportional to the heat-kernel coefficient, $C_{1}$, and equals,

$$
\begin{aligned}
F_{3}(T, \beta) & =-\frac{\beta}{8 \pi^{(d+3) / 2}} \zeta_{R}(d-1) B_{2}^{(d)}\left((d-1) / 2 \mid \frac{\pi}{\beta}, \mathbf{1}\right) T^{d-1} \\
& =-\frac{2^{d-3}}{6 \sqrt{\pi}} \zeta_{R}(d-1)\left(\frac{\pi}{\beta}-\frac{\beta}{2 \pi}(d-1)\right) T^{d-1} .
\end{aligned}
$$

The subtracted quantity, (1), constructed from (11) always has a maximum at $\beta=\pi$, whatever the dimension, $d$. (I am again setting $\alpha=2 \pi$ for convenience.) This is a limiting behaviour, and to obtain the precise dependency a numerical evaluation is required. This would yield the value of the temperature at which any central minimum just turns into a maximum ${ }^{4}$. The finite temperature correction (the second term in (10)) is always negative and has a maximum at the mid point. The value of $F$ is a competition between the two parts of (10).

As the walls are approached, the zero temperature part of the free energy, (10), dominates, again giving a repulsion for the odd dimensions, $3,7, \ldots$. The graph of

\footnotetext{
${ }^{4}$ It is really the value of the dimensionless quantity, $a T$, that is determined, where $a$ is the radius of the lune. For $\alpha=2 \pi$ the value of this transition temperature is approximately 0.455 for Neumann conditions in three dimensions.
} 
the free energy against the angle of the piston is that of a double well potential so that the piston, this time, would preferentially settle in one of the two minima symmetric about the mid point, a somewhat curious situation, if it could be realised. For $T=0.6$ these minima are very approximately at $\beta=\pi / 2$ and $\beta=3 \pi / 2$. In even dimensions, a finite temperature provides an attraction to the walls.

For the record, I give here the first three terms in the high temperature expansion of the free energy on a 3 -lune of angle $\alpha$,

$$
F(T, \alpha) \approx-\frac{\pi \alpha}{90} T^{4}-\frac{\zeta_{R}(3)}{4} T^{3}-\frac{\pi^{3 / 2}}{36}\left(\frac{\pi}{\alpha}-\frac{\alpha}{\pi}\right) T^{2}-
$$

which is useful when checking the exact form, (10).

\section{Conclusion}

In the 'physical' dimension, $d=3$, the hemispherical piston arrangement is stable and would oscillate about the midpoint, if the temperature were below the transition value. For higher temperatures, the equilibrium position would shift to one of two minima symmetrically placed with respect to the midpoint.

By contrast, for $d=5$ say, the system is unstable, and the piston would collapse to one of the walls, whatever the temperature.

It seems difficult to maintain that the system analysed in this paper is a practical one. Rather, it should be considered as a mathematical fiction whose value lies in a relatively easy analysis.

It has to be admitted that this simplicity is, to some extent, accidental, being a result of the conformal propagation equations which allow $\zeta_{\mathcal{M}}(-1 / 2)$ to be finite. If these are modified, say by adding a mass, then divergences associated with conical singularities appear which are not removed by the subtraction, (9), nor, worse, are not independent of the position of the piston. (See Appendix 2.) That this does not occur in the rectangular piston system is a consequence of the fact that there the constituents are always perpendicular. If the piston were allowed to slant, even slightly, not only would the force change but it would acquire a divergence, which raises questions of principle.

The system described in the present paper is a curved space analogue of the traditional wedge geometry investigated over the years. Most discussions involve the energy density, but there are some of the total Casimir energy in a bounded wedge. Recent calculations that make relevant remarks on divergences in the light of various 
boundary conditions are Milton, Wagner and Kirsten, [14], Brevik, Ellingsen and Milton, [15] and Ellingsen, Brevik and Milton, [16].

In order to more closely mimic the wedge bounded by a circular arc in flat space, it is possible to impose boundary conditions at the equator of the $d$-sphere which produces half a $d$-lune. The eigenvalues are a simple modification of (4) and details will be exposed at another time.

\section{Appendix 1. Alternating extrema}

I prove that the vacuum energy, (9), is a maximum or a minimum at the mid point depending on the sign $(-1)^{(d+1) / 2}$, for odd $d$. I do this by computing the second derivative of $E(\beta, \alpha)$ with respect to $\beta$ at $\beta=\alpha / 2$.

There is nothing special about any particular value of the outer wall angle, $\alpha$, which I now set equal to $2 \pi$. The mid point is then at $\beta=\pi$, i.e. $q=1$, its most convenient value.

At the mid point, the first derivatives of the first two terms in (9) cancel, while the second derivatives are equal, and add. Hence it is sufficient to evaluate, using (6)

$$
-\frac{\partial^{2}}{\partial x^{2}}\left(x B_{d+1}^{(d)}\left((d-1) / 2 \mid \frac{1}{x}, \mathbf{1}\right)\right)
$$

at $x=1$ where $x=\beta / \pi$. In order to more directly employ Barnes' formula, (7), I use the variable $q=1 / x$ and therefore seek,

$$
-q^{2} \frac{\partial}{\partial q} q^{2} \frac{\partial}{\partial q} \frac{1}{q} B_{\nu}^{(d)}(a \mid q, \boldsymbol{\omega})=q^{2} \frac{\partial}{\partial q} B_{\nu}^{(d+1)}(a+q \mid q, q, \boldsymbol{\omega}),
$$

for the special values, $\nu=d+1, a=(d-1) / 2, q=1$ and $\boldsymbol{\omega}=\mathbf{1}$.

The three dependencies on $q$ on the right-hand side of (13) are treated separately, and, using the symmetry of the parameters $\left(q, q^{\prime}, \boldsymbol{\omega}\right)$ in $q$ and $q^{\prime}$, I find the general formula,

$$
\begin{aligned}
\frac{\partial}{\partial q} B_{\nu}^{(d+1)}(a+q \mid q, q, \boldsymbol{\omega}) & = \\
\left.2 \frac{\partial}{\partial q} B_{\nu}^{(d+1)}\left(a \mid q, q^{\prime}, \boldsymbol{\omega}\right)\right|_{\substack{a=a+q \\
q^{\prime}=q}}+\left.\frac{\partial}{\partial a} B_{\nu}^{(d+1)}(a \mid q, q, \boldsymbol{\omega})\right|_{a=a+q} & = \\
-\frac{2}{q} B_{\nu}^{(d+2)}(a+2 q \mid q, q, q, \boldsymbol{\omega})+\frac{2}{q} B_{\nu}^{(d+1)}(a+q \mid q, q, \boldsymbol{\omega}) & \\
& +\nu B_{\nu-1}^{(d+1)}(a+q \mid q, q, \boldsymbol{\omega}),
\end{aligned}
$$


where I have used (7) again, and a standard identity for Bernoulli polynomials.

Inserting the special values, it is seen, firstly, that the Bernoulli polynomials have all their parameters equal to unity, the most extensively discussed case. Secondly, for $d$ odd, the arguments $a+2 q=(d+3) / 2$ and $a+q=(d+1) / 2$ are positive integers and, furthermore, are such as to make the first and last terms on the final line vanish. This is because of the product structure (e.g. [17] p.186, [18], §8),

$$
B_{n}^{(n+1)}(x \mid \mathbf{1})=(x-1)(x-2) \ldots(x-n),
$$

which always possesses a vanishing factor. This leaves the second term, whose contribution to the second derivative at the mid point is

$$
4 B_{d+1}^{(d+1)}((d+1) / 2 \mid \mathbf{1})
$$

and it is the sign of this quantity that I require. I use the representation

$$
B_{n}^{(n)}(x \mid \mathbf{1})=\int_{x}^{x+1} d t(t-1)(t-2) \ldots(t-n)
$$

and obtain, after translating the integration variable,

$$
B_{d+1}^{(d+1)}((d+1) / 2 \mid \mathbf{1})=\int_{-1 / 2}^{1 / 2} d t\left(t^{2}-\left(\frac{1}{2}\right)^{2}\right) \ldots\left(t^{2}-\left(\frac{d}{2}\right)^{2}\right) .
$$

It is easy to see that in the integration range, $-1 / 2 \leq t \leq 1 / 2$, the integrand is positive for $d=3,7,11, \ldots$ and negative for $d=5,9,13, \ldots$. This means that the vacuum energy is a minimum at the mid point in the former cases and a maximum in the latter, which was to be proved.

\section{Appendix 2. Divergences for non-conformal coupling}

To illustrate the emergence and character of the divergences, it is sufficient to add a small mass term, $\delta m^{2}$, to the conformal eigenvalues $\lambda_{n}$, (4), and employ a binomial, perturbation expansion to give the resulting change in the $\zeta$-function,

$$
\delta \zeta_{d}(s, a \mid \boldsymbol{\omega})=-\delta m^{2} s \zeta_{d}(2 s+2, a \mid \boldsymbol{\omega})
$$

which. with the appropriate $a$ and $\boldsymbol{\omega}$, should be added to the conformal $\zeta$-functions, (5). The correction, (15), possesses a pole at the evaluation point $s=-1 / 2$ with residue proportional to the Bernoulli polynomial,

$$
B_{d-1}^{(d)}((d-1) / 2 \mid \pi / \beta, \mathbf{1})
$$


examples of which are the well known forms, associated with conical singularities,

$$
\begin{aligned}
& \frac{\pi^{2}-\beta^{2}}{6 \beta^{2}}, \quad d=3 \\
& -\frac{\left(\pi^{2}-\beta^{2}\right)\left(\pi^{2}+11 \beta^{2}\right)}{30 \beta^{4}}, \quad d=5,
\end{aligned}
$$

and are, up to a factor, the heat-kernel coefficients, $C_{(d-1) / 2}$, on the lune. For even $d$, the Bernoulli polynomial is always proportional to just $\pi / \beta$.

These results show that the $\beta$-dependence of the divergence does not disappear from the vacuum energy combination (9). This presents us with an old problem of interpretation which is not resolved here. For this reason I do not take (15) any further. (Even though the number of pole terms is finite for any given $d$, when the higher perturbation terms are evaluated.) 


\section{References.}

1. Cavalcanti,R.M. Phys. Rev. D69 (2004) 065015.

2. Kirsten,K. and Fulling,S.A. Phys. Rev. D79 (2009) 065019 .

3. Fucci and Kirsten,K, The Casimir Effect for Conical Pistons, ArXiv:1101.5409.

4. Dowker,J.S. and Kennedy,G. J. Phys. A (1978) 895.

5. Lukosz,W, Physica 56 (1971) 109; Z. f. Phys. 258 (1973) 99 ;Z. f. Phys. 262 (1973) 327.

6. Dowker,J.S. and Banach,R. J. Phys. A11 (1978) 2255.

7. Gromes, D. Math. Zeit. 94 (1966) 110.

8. Pockels, F. Über die Differentialgleichung $\Delta u+k^{2} u=0$ (Teubner, Leipzig. 1891).

9. Chang,P. and Dowker,J.S. Nucl. Phys. B395 (1993) 407.

10. Barnes,E.W. Trans. Camb. Phil. Soc. 19 (1903) 374.

11. Dowker.J.S. Nucl.Phys.B (Proc.Suppl) (104)(2002)153; also Dowker,J.S. hepth/ 0007129.

12. Fulling,S.A, Kaplan L. and Wilson,J.H. Phys. Rev. A76 (2007) 012118.

13. Dowker,J.S. Class. Quant. Grav. 1 (1984) 359.

14. Milton, K.A., Wagner,J. and Kirsten,K. Phys. Rev. D80 (2009) 125028.

15. Ellingsen,S.A.,Brevik,I. and Milton,K.A. Phys. Rev. E80 (2009) 021125.

16. Brevik,I., Ellingsen,S.A. and Milton,K.A. Phys. Rev. E79 (2009) 041120.

17. Nörlund,N.E. Acta Mathematica 43 (1922) 121.

18. Barnes,E.W. Trans. Camb. Phil. Soc. 19 (1903) 426. 